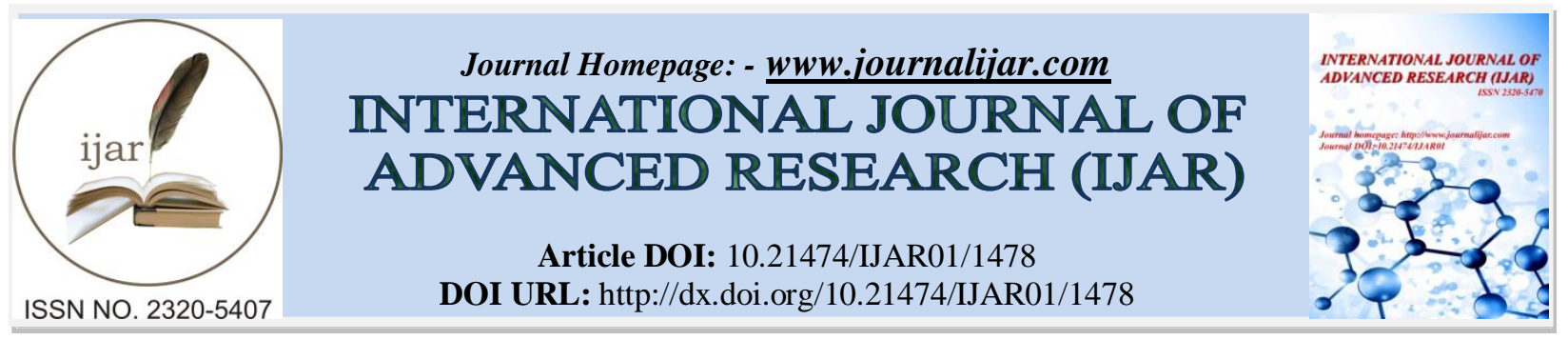

RESEARCH ARTICLE

\title{
DESTROYER PATHOGEN OF POTATO (SOLANUM TUBEROSUM) IN GEORGIA.
}

\begin{abstract}
O. Shainidze*, Sh. Lamparadze, A. Murvanidze and J. Diasamidze.
Batumi Shota Rustaveli State University, Faculty of Technology, Department of Agroecology and Plant protection, Ninoshvili str. 35, Batumi, 6010 Georgia.
\end{abstract}

\section{Manuscript Info}

Manuscript History

Received: 12 July 2016

Final Accepted: 12 August 2016

Published: September 2016

Key words:-

Phytophthora infestans, Late blight,

Development, Spread.

\section{Abstract}

Phytophthora infestans (Oomycota) remains a problem to production agriculture. Historically there have been many controversies concerning its biology and pathogenicity, some of which remain today. Composition pathogenic fungi potato (Solanum tuberosum) is discussed in this article. 50 species of microbiota fully identified. Also, 11 species of fungi involved in the formation of the consortium on tuber potato plants were identified. Long-term (1985-2015) monitoring showed that the destructive pathogen potato late blight is what causes the most damage and economic losses. favorable and unfavorable weather conditions and the development of its distribution was found. Experiments have shown that widespread rot of potato is significantly different from other types of fungi in the aggressiveness and severity. He can completely destroy all the varieties of potato plants in some localities within 5-6 days. The highest disease severity was on the Nevsky and Picaso variety, the highest resistance to diseases manifested in 3 years German variety - Kardena,Russian Sante, Finland - Katu and Holland variety - Desire.

Copy Right, IJAR, 2016,. All rights reserved.

\section{Introduction:-}

Potatoes (Solanaceae) are a staple food and occupy a prominent place in many national cuisines. He almost complete food product, providing all the necessary nutrients.

Potatoes has a worldwide increasing value (Birch et al., 2012) and considered to be the fourth most important food crop (wheat, maize and rice), is cultivated in temperate and subtropical regions across the world.

In Georgia, there have been significant advances in food production over the past six decades, in connection with the adoption of improved techniques, including high-yielding varieties of potatoes. Unfortunately, potato production is constrained primarily due to biotic and abiotic stresses. Among the biotic factors of Phytophthora infestans (potato late blight) remains a challenge for production in agriculture.

Potato late blight caused by the fungus Phytophthora infestans (Mont.) de Bary (Oomycetes) is one of the most important diseases of potato (Solanum tuherosum). Leaves and tubers of susceptible cultivars become readily infected by this pathogen. The fungus spreads rapidly through the plant tissue, causing a destructive necrosis.

Corresponding Author:- O. Shainidze.

Address:- Batumi Shota Rustaveli State University, Faculty of Technology, Department of Agroecology and Plant protection, Ninoshvili str. 35, Batumi, 6010 Georgia. 
Late blight was a major culprit in the 1840s European, the 1845 Irish and 1846 Highland potato famines. The organism can also infect tomatoes and some other members of the Solanaceae. In the world, this disease causes about $\$ 6$ billion damage to crops each year (Chand, Sudeep, 2009; Nowicki, Marcin; et al., 2011, 2013). Direct cash costs of efforts to control and production losses are estimated only Potata> \$ 3 billion / year worldwide CIP, 1996). In this regard, at least, have a bad situation in Georgia. In the Georgian Republic under favorable cold and wet conditions and without any corresponding levels of regulation against the pathogen, P. infestans can lead to almost $100 \%$ reduction in crop yields; therefore, potatoes can not be controlled without frequent fungicide applications for the effective control of the disease.

Even now, more than 170 years after it was first associated with the potato late blight disease in Europe and in North America, P. infestans remains a major problem in agriculture and recalcitrant to low-input, stable disease suppression.

Despite the fact that considerable progress has been made in the understanding of its basic biology, ecology and pathogenicity (Coffey, Wilson, 1983; Bourke,1991; Andrivon, 1996; Fay and Fry, 1997; Forbes et al.,1997; Turkensteen et al., 2000; Smart et al, 2000; Vleeshouwers et al., 2000; Aylor et al., 2001; Garrett et al., 2001; Lee et al., 2002; Fabritius et al, 2002; Grunwald et al., 2002b; Judelson and Roberts, 2002; Cvitanich and Judelson, 2003a, 2003b; Madden and Wheelis, 2003; Madden and Wheelis, 2003; Stewart et al, 2003; Fernandez-Pavia et al, 2004; Latijnhouwers et al., 2004; Grunwald and Flier, 2005; Whisson et al., 2005; Tani and Judelson, 2006; Mizubuti and Fry, 2006; Flier et al, 2007; Whisson et al., 2007; Widmark et al., 2007), and there are a lot of books (Dowley et al., 1995), thousands of scientific articles and thousands of popular reports, as well as many historical processes (Turner, 2005); etc., P. infestans is still a serious problem in agriculture. In Georgia, a considerable amount of research has been devoted to date on various aspects of relations P. infestans potato (Kanchaveli, 1978I; Shainidze, 1999, 2000, 2013; and et al.). Here we look at the current state of the patogena.

\section{Research objectives:-}

The objectives of this study was to identify and determine the composition of pathogenic fungi that propagate in the Solanum tuberosum weight agrocenoses Georgia; among them to clarify and examine the most common potato destructor; to establish the scope of its development - the spread under favorable and enabling environment. In addition, the aim of this study was to evaluate the resistance of varieties of potatoes Phytophtora infestans in relation to the effectiveness and stable yields.

\section{Materials and methods:-}

The object of the study were different varieties of potato plants (Solanum tuberosum) and pathogenic fungi which inhabit agrocenoses weight Georgia. Materials were collected using well known methods (Bilai et al, 1982; Giants et al, 1980; Dudka et al, 1982; Foster et al., 2004). Routing and stationary methods were used to study the field of research. Symptoms such as rot, mummification, wilting, spotting, necrosis, mold, Gauls, ulcer, deformation, chlorosis, mosaic, etc., were recorded. The study upper and lower parts of infected plants were collected and labeled. The following procedures, such as off-site and laboratory treatment, collection of preserved plant specimens, assembly, storage, assessment of the infected plants have been carried out for processing the collected samples. We analyzed the distribution and development of disease. Identification of fungi was carried out using modern identification guides (Hawksworth, 1974; Khokhryakov et al, 1984; Watanabe, 2000).

Collections of the new species have been examined by standard light microscopy (Pereval, Carl Zeiss, Jena and Olympus, BX 50, Hamburg,Germany). The SEM micrographs have been prepared by means of a JSM-35 (Japan) SEM microscope. The specimens examined are deposited at HAL, KW and TGM (Holmgren et al., 1990). Collections were examined by standard light microscopy (Pass, Carl Zeiss, Jena, and Olympus, BX 50, Hamburg, Germany). SEM photomicrographs were prepared using DSM-35 (Japan) SEM microscope. Samples were deposited on Hal, KW and TGM (Holmgren et al., 1990).

\section{Results and Discussion:-}

A total of 50 species (including 13 species of virus and viroid, 5 - bacteria, 32 - the fungus) have been identified in Solanum tuberosum (Tabel 1), as a result of phytopathological and mycological studies carried out in different places (agrocenosis Georgia) in industrial regions. Among the total microbiota, late blight is one of the most devastating potato diseases Sometimes, during storage, infected tubers may be covered with different colored mycelia (Figure 1), which includes 11 species of fungi (Alternaria solani, Aspergillus Niger, Botritis cinerea, 
Fusarium solani, F. moniliforme, Mucor sp., Penicillium citrinum, Phytophtora infestans, Sclerotium rolfsi, Rhizoctonia solani, Rhizopus psapzzh). Infected tubers are often invaded by soft rot bacteria which rapidly convert adjoining healthy potatoes into a smelly, rotten mass that must be discarded.

Some are plant pathogens causing root and stem rot, vascular wilt or fruit rot. Other species cause storage rot and are important mycotoxin producers. Several species, notably Fusarium moniliforme, F. oxysporum, and F. solani, are recognized as being pathogenic to man and animals (Kriek, Kellerman and Marasas,1981; Kriek, Marasas and Thiel, 1981).

It should be noted that a wide range of microbiota tubers (Consortium, Association) marks the first time in Georgia (maybe all over the world).

Observations have shown that the formation of a consortium of home begins when the temperature reaches $-20^{\circ} \mathrm{C}$, and the optimum temperature is about $22{ }^{\circ} \mathrm{C}$. High air humidity (90-95\%) accelerates the formation of the consortium. Currently, research is ongoing to discover the initiator of the fungus, taking part in the creation of the consortium and to determine the relationship between the fungi involved in it. Common symptom of the disease is the "Leaf Blight", which is found everywhere.

Lesions on leaf blades can be extended to the shell sheet. Defeat increases in length and width, and may also have wavy edges.

Long-term monitoring showed that in Georgia potato rot is identified by black or brown lesions (Figure 2) on the leaves and stems, which at first glance it may seem that small and watery or have chlorotic borders, but soon quickly expand and become necrotic.

Infected tubers occur when sporangia are washed from the leaves into the soil. Infections generally begin in tuber cracks, eyes or lenticels.

Tabel 1:- Systematical structure of microbiota Potato.

\begin{tabular}{|c|c|c|c|c|c|c|}
\hline $\begin{array}{l}\text { Microbiota } \\
\text { (Exciter) }\end{array}$ & Division & Class & Order & Family & Genus & Species \\
\hline Rhizaria & Cercozoa & Phytomyxea & $\begin{array}{l}\text { Plasmodiopho- } \\
\text { rales }\end{array}$ & $\begin{array}{l}\text { Plasmodiophora- } \\
\text { ceae }\end{array}$ & Spongospora & $\begin{array}{l}\text { Spongospora } \\
\text { subterranea }\end{array}$ \\
\hline \multirow[t]{4}{*}{ Chromista } & \multirow[t]{4}{*}{ Oomycota } & \multirow[t]{4}{*}{ Oomycetes } & \multirow{4}{*}{ Peronosporales } & Pythiaceae & Pythium & $\begin{array}{l}\text { Pythium } \\
\text { deliense }\end{array}$ \\
\hline & & & & Phytophthoraceae & Phytophthora & $\begin{array}{l}\text { Phytophthora } \\
\text { cryptogea }\end{array}$ \\
\hline & & & & & & $\begin{array}{l}\text { Phytophthora } \\
\text { drechsleri }\end{array}$ \\
\hline & & & & & & $\begin{array}{l}\text { Phytophthora } \\
\text { infestans }\end{array}$ \\
\hline \multirow[t]{7}{*}{ Fungi } & $\begin{array}{l}\text { Chytridiomy- } \\
\text { cota }\end{array}$ & $\begin{array}{l}\text { Chytridiomy- } \\
\text { cete }\end{array}$ & Synchytriales & Synchytriaceae & Synchytrium & $\begin{array}{l}\text { Synchytrium } \\
\text { endobioticum }\end{array}$ \\
\hline & \multirow[t]{2}{*}{ Zygomycota } & \multirow{2}{*}{$\begin{array}{l}\text { Mucormyco- } \\
\text { tina }\end{array}$} & \multirow[t]{2}{*}{ Mucorales } & \multirow[t]{2}{*}{ Mucoraceae } & Mucor & Mucor sp. \\
\hline & & & & & Rhizopus & $\begin{array}{l}\text { Rhizopus } \\
\text { nigricans }\end{array}$ \\
\hline & \multirow[t]{4}{*}{ Ascomycota } & $\begin{array}{l}\text { Sordariomyc- } \\
\text { etes }\end{array}$ & Hypocreales & Nectriaceae & Gibberella & $\begin{array}{l}\text { Gibberella } \\
\text { pulicaris } \\
\text { (Fusarium } \\
\text { solani) }\end{array}$ \\
\hline & & & & & & $\begin{array}{l}\text { Gibberella } \\
\text { acuminatum }\end{array}$ \\
\hline & & $\begin{array}{l}\text { Leotiomyce- } \\
\text { tes }\end{array}$ & Helotiales & Sclerotiniaceae & Botryotinia & $\begin{array}{l}\text { Botryotinia } \\
\text { fuckeliana }\end{array}$ \\
\hline & & Dothideomy- & Capnodiales & Mycosphaerellac & Mycovellosiella & Mycovellosiella \\
\hline
\end{tabular}




\begin{tabular}{|c|c|c|c|c|c|c|}
\hline & & cetes & & eae & & concors \\
\hline & & & & & Cercospora & $\begin{array}{l}\text { Cercospora } \\
\text { solani- tuberosi }\end{array}$ \\
\hline & & Ascomycetes & Incertae sedis & Incertae sedis & Polyscytalum & $\begin{array}{l}\text { Polyscytalum } \\
\text { pustulans }\end{array}$ \\
\hline & & $\begin{array}{l}\text { Leotiomyce- } \\
\text { tes }\end{array}$ & Helotiales & Sclerotiniaceae & Sclerotinia & $\begin{array}{l}\text { Sclerotinia } \\
\text { sclerotiorum }\end{array}$ \\
\hline & & $\begin{array}{l}\text { Eurotiomyce- } \\
\text { tes }\end{array}$ & Eurotiales & Trichocomaceae & Aspergillus & $\begin{array}{l}\text { Aspergillus } \\
\text { niger }\end{array}$ \\
\hline & & & & & Penicillium & $\begin{array}{l}\text { Penicillium } \\
\text { citrinum }\end{array}$ \\
\hline & & $\begin{array}{l}\text { Leotiomyce- } \\
\text { tes }\end{array}$ & Helotiales & Sclerotiniaceae & Botrytis & Botrytis cinerea \\
\hline & & $\begin{array}{l}\text { Dothideomy- } \\
\text { cetes }\end{array}$ & Pleosporales & Pleosporaceae & Alternaria & $\begin{array}{l}\text { Alternaria } \\
\text { alternata }\end{array}$ \\
\hline & & & & & & $\begin{array}{l}\text { Alternaria } \\
\text { solani }\end{array}$ \\
\hline & & Sordariomy- & Hypocreales & Nectriaceae & Fusarium & $\begin{array}{l}\text { Fusarium } \\
\text { equiseta }\end{array}$ \\
\hline & & cetes & & & & $\begin{array}{l}\text { Fusarium } \\
\text { crookwellense }\end{array}$ \\
\hline & & $\begin{array}{l}\text { Sordariomyc } \\
\text { etes }\end{array}$ & Hypocreales & Incertae sedis & Verticillium & $\begin{array}{l}\text { Verticillium } \\
\text { albo-atrum }\end{array}$ \\
\hline & & & & & & $\begin{array}{l}\text { Verticillium } \\
\text { dahlia }\end{array}$ \\
\hline & & $\begin{array}{l}\text { Dothideomy- } \\
\text { cetes }\end{array}$ & Pleosporales & Pleosporaceae & $\begin{array}{l}\text { Helminthospori- } \\
\text { um }\end{array}$ & $\begin{array}{l}\text { Helminthospori } \\
\text { um solan }\end{array}$ \\
\hline & & $\begin{array}{l}\text { Sordariomy- } \\
\text { cetes }\end{array}$ & Glomerellales & Glomerellaceae & Colletotrichum & $\begin{array}{l}\text { Colletotrichum } \\
\text { atramentarium }\end{array}$ \\
\hline & & & & & & $\begin{array}{l}\text { Colletotrichum } \\
\text { coccodes }\end{array}$ \\
\hline & & $\begin{array}{l}\text { Dothideomy- } \\
\text { cetes }\end{array}$ & Capnodiales & $\begin{array}{l}\text { Mycosphaerella- } \\
\text { ceae }\end{array}$ & Septoria & $\begin{array}{l}\text { Septoria } \\
\text { lycopersici var. } \\
\text { malagutii }\end{array}$ \\
\hline & & $\begin{array}{l}\text { Dothideomy- } \\
\text { cetes }\end{array}$ & $\begin{array}{l}\text { Botryosphaeri- } \\
\text { ales }\end{array}$ & $\begin{array}{l}\text { Botryosphaeria- } \\
\text { ceae }\end{array}$ & Macrophomina & $\begin{array}{l}\text { Macrophomina } \\
\text { phaseolina }\end{array}$ \\
\hline & & $\begin{array}{l}\text { Dothideomy- } \\
\text { cetes }\end{array}$ & Pleosporales & Incertae sedis & Phoma & $\begin{array}{l}\text { Phoma solani- } \\
\text { cola f. foveata }\end{array}$ \\
\hline & $\begin{array}{l}\text { Basidiomyc- } \\
\text { ota }\end{array}$ & $\begin{array}{l}\text { Agaricomy- } \\
\text { cetes }\end{array}$ & Cantharellales & $\begin{array}{l}\text { Ceratobasidiace- } \\
\text { ae }\end{array}$ & Rhizoctonia & $\begin{array}{l}\text { Rhizoctonia } \\
\text { solani }\end{array}$ \\
\hline & & & Atheliales & Atheliaceae & Athelia & $\begin{array}{l}\text { Athelia rolfsii } \\
\text { anamorph } \\
\text { (Sclerotium } \\
\text { rolfsii) }\end{array}$ \\
\hline Bacteria & $\begin{array}{l}\text { Proteobacte- } \\
\text { ria }\end{array}$ & $\begin{array}{l}\text { Beta Proteo- } \\
\text { bacteria }\end{array}$ & Burkholderiales & Ralstoniaceae & Ralstonia & $\begin{array}{l}\text { Ralstonia } \\
\text { solanacearum }\end{array}$ \\
\hline & & $\begin{array}{l}\text { Gamma } \\
\text { proteobac- }\end{array}$ & Enterobacteria- & Enterobacteria- & Pectobacterium & $\begin{array}{l}\text { Pectobacterium } \\
\text { atrosepticum }\end{array}$ \\
\hline & & teria & les & ceae & & $\begin{array}{l}\text { Pectobacterium } \\
\text { carotovorum } \\
\text { Syn.:Erwinia } \\
\text { carotovora }\end{array}$ \\
\hline & $\begin{array}{l}\text { Actinobacte- } \\
\text { ria }\end{array}$ & $\begin{array}{l}\text { Actinobacte- } \\
\text { ria }\end{array}$ & $\begin{array}{l}\text { Actinomyceta- } \\
\text { les }\end{array}$ & $\begin{array}{l}\text { Microbacteria- } \\
\text { ceae }\end{array}$ & Clavibacter & $\begin{array}{l}\text { Clavibacter } \\
\text { michiganensis }\end{array}$ \\
\hline
\end{tabular}




\begin{tabular}{|c|c|c|c|c|c|c|}
\hline & & & & $\begin{array}{l}\text { Streptomycetac- } \\
\text { eae }\end{array}$ & Streptomyces & $\begin{array}{l}\text { Streptomyces } \\
\text { scabiei }\end{array}$ \\
\hline \multirow[t]{13}{*}{$\begin{array}{l}\text { Virus and } \\
\text { viroid }\end{array}$} & & - & $\begin{array}{l}\text { Group } \\
\text { IV }((+) \text { ssRNA })\end{array}$ & Potyviridae & Potyvirus & $\begin{array}{l}\text { Potato virus A } \\
\text { (PVA) }\end{array}$ \\
\hline & & $\begin{array}{l}\text { Group } \\
\text { IV }((+) \\
\text { ssRNA })\end{array}$ & Tymovirales & Betaflexiviridae & Carlavirus & $\begin{array}{l}\text { Potato virus M } \\
(\mathrm{PVM})\end{array}$ \\
\hline & & $\begin{array}{l}\text { Group } \\
\text { IV }((+) \operatorname{ssRN} \\
\text { A) }\end{array}$ & Tymovirales & Betaflexiviridae & Carlavirus & $\begin{array}{lll}\text { Potato virus } & \text { S } \\
\text { (PVS) } & & \end{array}$ \\
\hline & & $\begin{array}{l}\text { Group } \\
\text { IV }((+) \text { ssRNA }\end{array}$ & Picornavirales & Secoviridae & Nepovirus & $\begin{array}{l}\text { Potato virus } \\
\text { (PVU) }\end{array}$ \\
\hline & & - & $\begin{array}{lr}\text { Group: } & \text { IV } \\
\text { (+)sense } & \text { RNA } \\
\text { Viruses } & \\
\end{array}$ & Potyviridae & Potyvirus & $\begin{array}{lll}\text { Potato } & \text { virus } & \text { V } \\
\text { (PVV) } & & \end{array}$ \\
\hline & & $\begin{array}{l}\text { Group } \\
\text { IV }((+) \text { ssRN } \\
\text { A) }\end{array}$ & Tymovirales & Alphaflexiviridae & Potexvirus & $\begin{array}{lll}\text { Potato } & \text { virus } & \mathrm{X} \\
(\mathrm{PVX}) & & \end{array}$ \\
\hline & & - & $\begin{array}{l}\text { Group:IV: } \\
\text { (+)sense RNA } \\
\text { Viruses }\end{array}$ & Potyviridae & Potyvirus & $\begin{array}{l}\text { Potato virus } \\
\text { (PVY) }\end{array}$ \\
\hline & & $\begin{array}{l}\text { Group } \\
\text { IV }((+) \text { ssRN } \\
\text { A) } \\
\end{array}$ & Tymovirales & Tymoviridae & Tymovirus & $\begin{array}{lr}\text { Andean } & \text { potato } \\
\text { latent } & \text { virus } \\
\text { (APLV) } & \\
\end{array}$ \\
\hline & & - & - & Comoviridae & Comovirus & 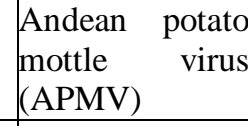 \\
\hline & & - & $\begin{array}{lr}\text { Group } & \text { IV: } \\
\text { (+)sense } & \text { RNA } \\
\text { Viruses } & \\
\end{array}$ & Comoviridae & Nepovirus & \begin{tabular}{|ll} 
Potato & black \\
ringspot & virus \\
(PBRSV) & \\
\end{tabular} \\
\hline & & $\begin{array}{l}\text { Group } \\
\text { IV }((+) \text { ssRN } \\
\text { A }\end{array}$ & Tymovirales & Betaflexiviridae & Carlavirus & $\begin{array}{l}\text { Potato latent } \\
\text { virus (PLV) }\end{array}$ \\
\hline & & - & $\begin{array}{l}\text { Group } \\
\text { IV }((+) \text { ssRNA })\end{array}$ & Luteoviridae & Polerovirus & $\begin{array}{l}\text { Potato leafroll } \\
\text { virus (PLRV) }\end{array}$ \\
\hline & & $\begin{array}{l}\text { (unranked): } \\
\text { Subviral } \\
\text { agents }\end{array}$ & $\begin{array}{l}\text { (unranked): } \\
\text { Viroid }\end{array}$ & Pospiviroidae & Pospiviroid & \begin{tabular}{|lr} 
Potato & spindle \\
tuber & viroid \\
$(P S T V d)$ & \\
\end{tabular} \\
\hline Total & 8 & 30 & 36 & 39 & 42 & 50 \\
\hline
\end{tabular}

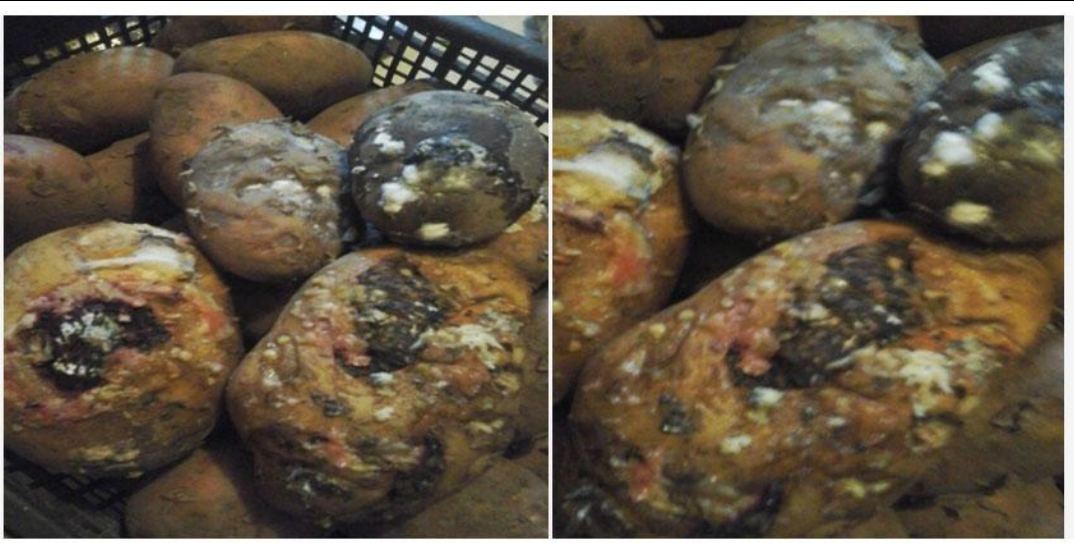

Figure 1:- Symptoms of consortium the Infected tubers. 
In humid conditions, late blight produces sporangia and sporangiophores on the surface of infected tissue. This leads to a significant increase in white sporulation at the forefront of defeats on the abaxial (lower) surfaces of the leaves and stems (Figure 3).

Sporangiophores grow out of diseased tissue. Sporangia are released into the atmosphere for aerial dispersal during a drop in relative humidity, or they can be dispersed in water splashes. Indirect germination releases zoospores, which, after encystment and germination on host tissue, produce lesions visible after 2- 4 days.
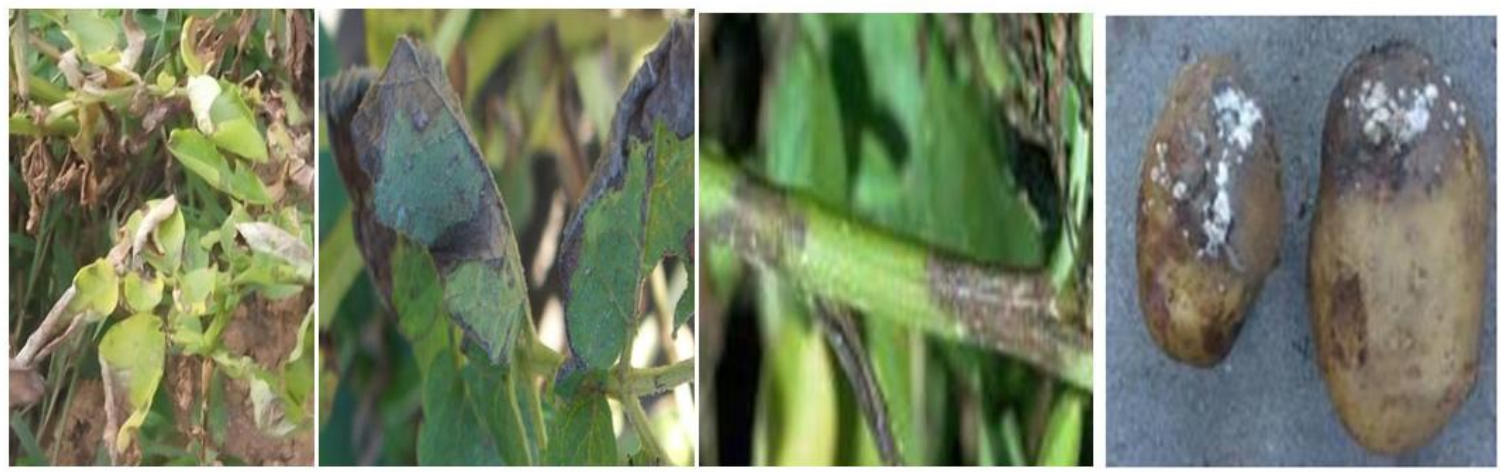

Figure 2:- Symptoms of late blight on the leaves, stems and tuberes of potato.

It has been found that in humid areas (Ajara) Georgia sporangia are formed, when the relative humidity is $89 \%$, and in mountainous areas - 90-91\%. Sporulation can occur from 3,5-26,5 ${ }^{\circ} \mathrm{C}$, but the optimum range is $18-22^{\circ} \mathrm{C}$. Sporangia germinate directly via a germ tube at $21-26^{\circ} \mathrm{C}$. Below $18^{\circ} \mathrm{C}$, sporangia produce 6 to 8 zoospores which require water for swimming.

Each zoospore is able to initiate an infection, which explains why the disease is more severe in cool, moist conditions.

After colonization leaf tissue Sporangiophores exit stomata thousands forming sporangia which can be moved by wind or rain, and eventually infect plants close or distant range. Almost similar findings were made by foreign researchers (Pristou and Gallegly, 1954; Gees and Hohl, 1988; Coffey and Wilson, 1983).

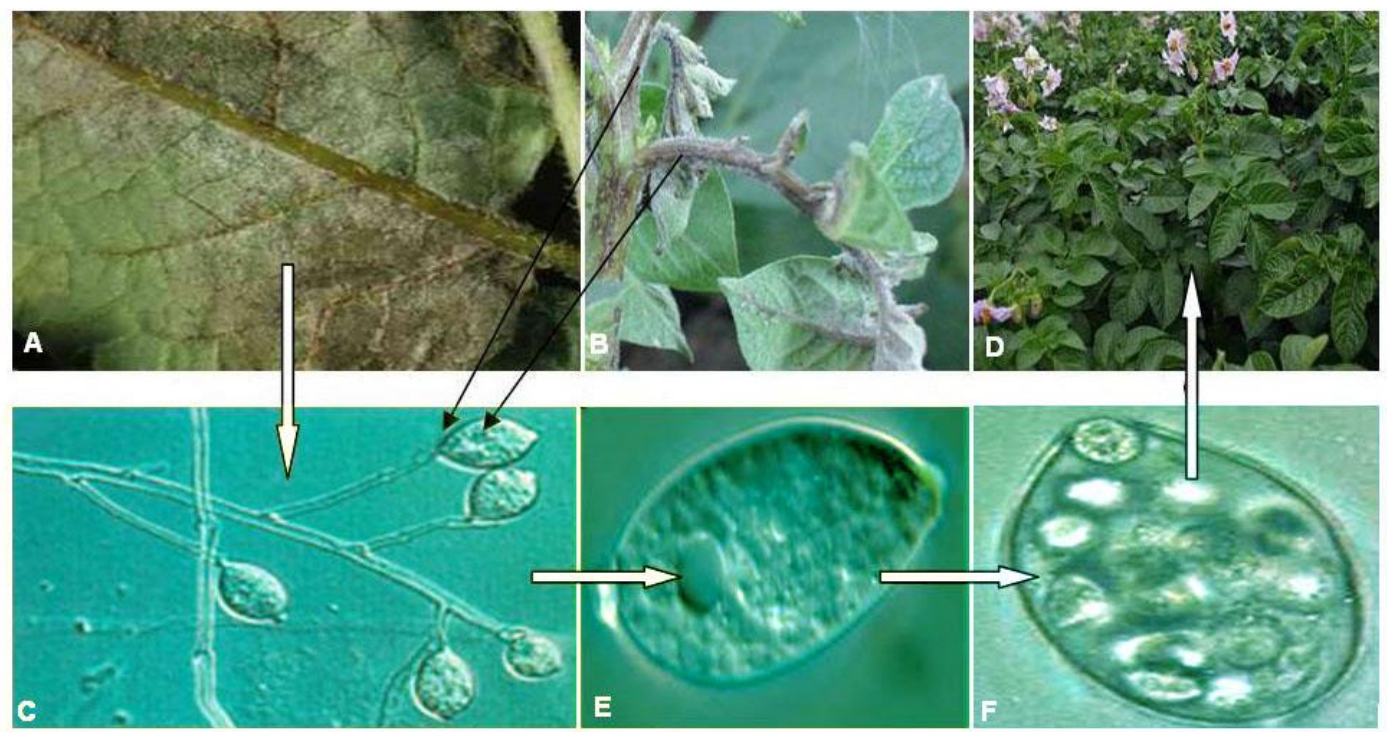

Figure 3:- Asexual life cycle of P. infestans: A,B,C - Sporangiophores grow out of diseased tissue; D - Sporangia are released into the atmosphere; $\mathrm{E}$ - Indirect germination zoospores, which produce lesions; F - visible after 2- 4 days. 
Solanum tuberosum as a host, the asexual life cycle of P. infestans can be completed rapidly with production of massive numbers of sporangia that are readily dispersed - explaining why whole fields can be transformed from slightly diseased to nearly completely destroyed within just a few days.

Late blight struck the growing plants as a frost in summer. It spread faster than other dangerous diseases.

The leaves, stems and tubers are all susceptible (Figure 4), so that the potato late blight pathogen, certainly deserves the name Phytophthora, "plant destroyer."

As a result of monitoring (2013-2015) it was established that late blight potato is characterized with different spread and intensity in different geographical zone.

Adjarian lowland is distinguished with high spreading of potato late blight (Diagram 1), where the spread of the disease is $92 \%$. Area under potato crops of Kvemo Kartli Plain is characterized with the lowest spread, where it reaches $44 \%$.

Investigations of areas under potato crops showed that inspite of sorts (Jelly, Europlant,Picasso, Marfona, Agria et al) diversity spreading late blight of potato fluctuates within 70-75 in The Samtskhe-Javakheti Region.

As spreading late blight of potato so intensity of disease development are comparatively high in Adjarian Lowland. Disease development intensity index in Adjara reaches 55\% (Figure 2). Intensity of development potato late blight is comparatively low 32-35\% in areas under potato crops of Samtskhe-Javakheti and Kvemo Kartli.

Intensity of spreading and development potato late blight according to regions was established by us. Results of monitoring showed that the high indicator spreading of late blight potato was fixed under potato plantations of Khulo, Shuakhevi, Keda, Khelvachauri and Kobuleti regions where spreading late blight potato reaches 87-92\%. Comparatively low spread of late blight potato (from 32-73\%) was fixed under potato plantations of Tsalka, Adigeni, Aspindza, Borjomi, Ninotsminda, Akhaltsikhe and Akalkalaki.

Late blight Potato is spread the least (30-39\%) in Bolnisi, Gardabani, Dmanisi, Marneuli and Tetritsqaro (Diagram 3).

Studies have shown that the intensity of the potato late blight development in general has been fixed at the average level for the regions (Figure 4).

Areas under of potato crops of Khulo and Shuakhevi are distinguished with comparatively high intensity of spreading potato late blight and disease development where the intensity reaches up to 53\%. Comparatively low indicator (31-34\%) Tsalka, Adigeni, Aspindza, Borjomi, Ninotsminda, Akhaltsikhe and Akalkalaki.

Intensity of spreading potato late blight is the lowest in areas under potato crop of Bolnisi, Gardabani, Dmanisi, Marneuli and Tetritsquaro. Its indicator is only from 26-30\%. These five regions are distinguished as with low indicator of spreading potato late blight so with the low frequency of development intensity. 


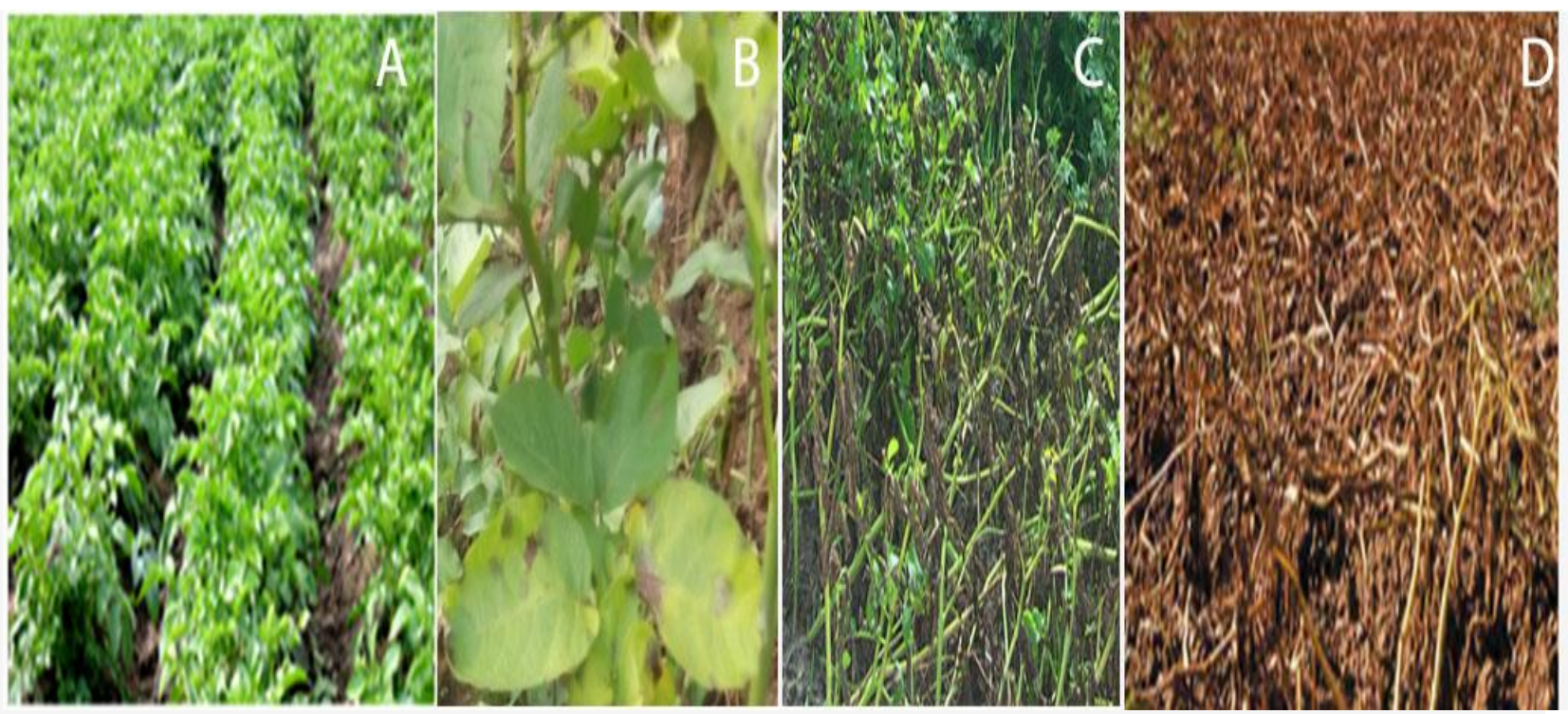

Figure 4:- The speed with which late blight can destroy a field of potatoes is impressive: A - healthy plant before flowering; B - siptomy affected leaves next week; C, D - and within another week can be totally destroyed

Diagram 1:- Spread of potato late blight according to zones in Georgia.

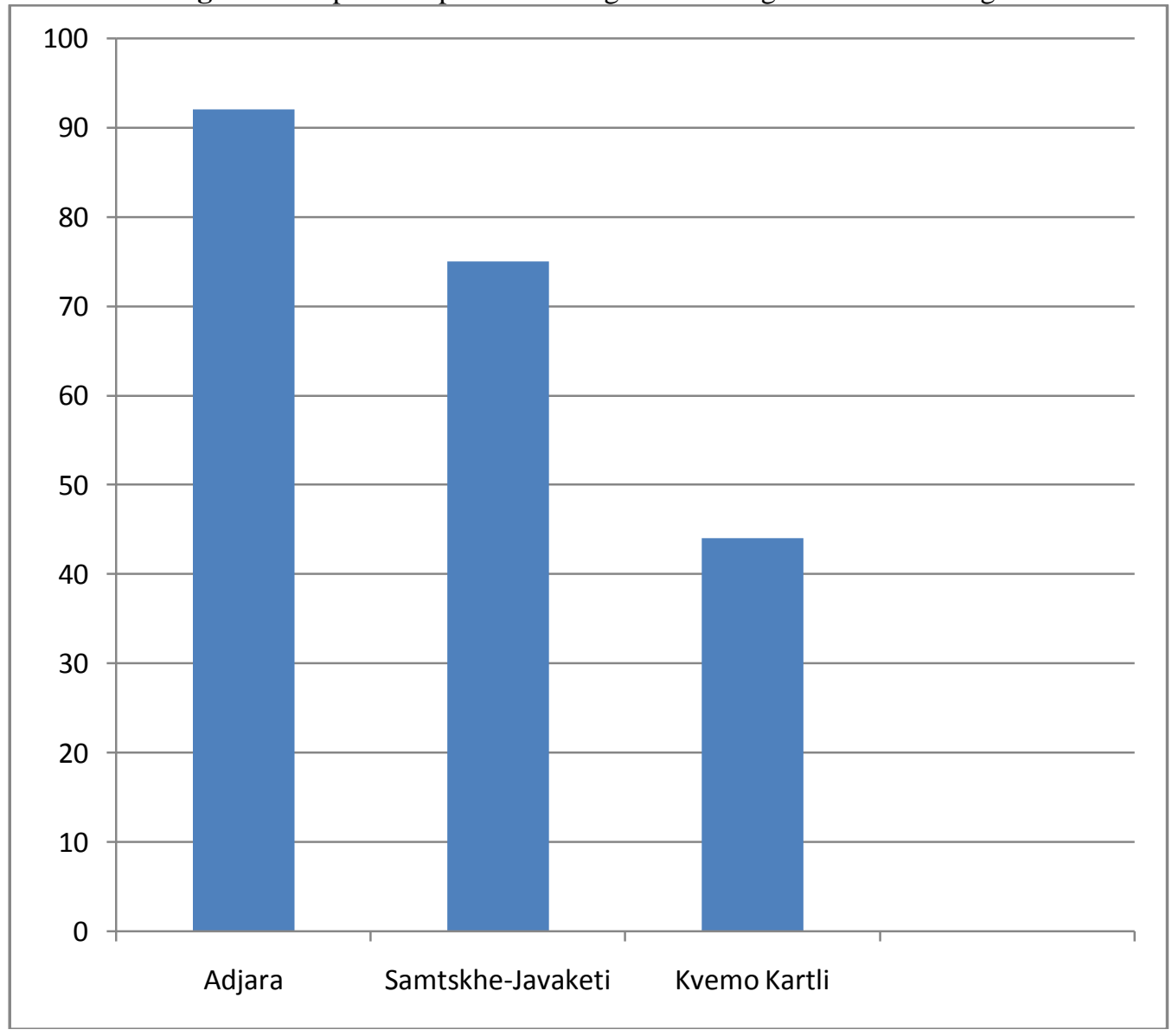


Diagram 2:- Intensity of development of late blight potato according to zones in Georgia

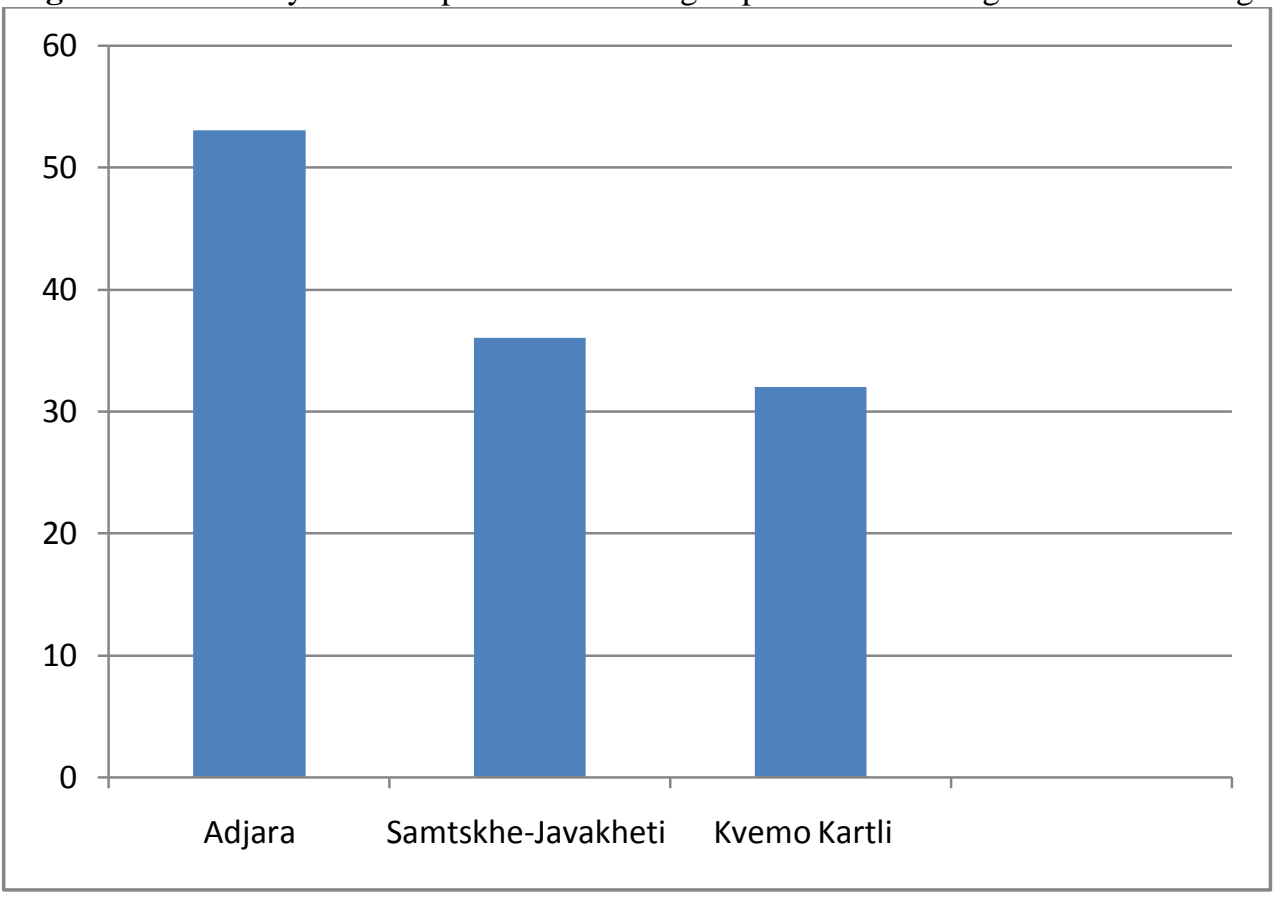

Diagram 3:- Spreading of potato late blight according to regions in Georgia

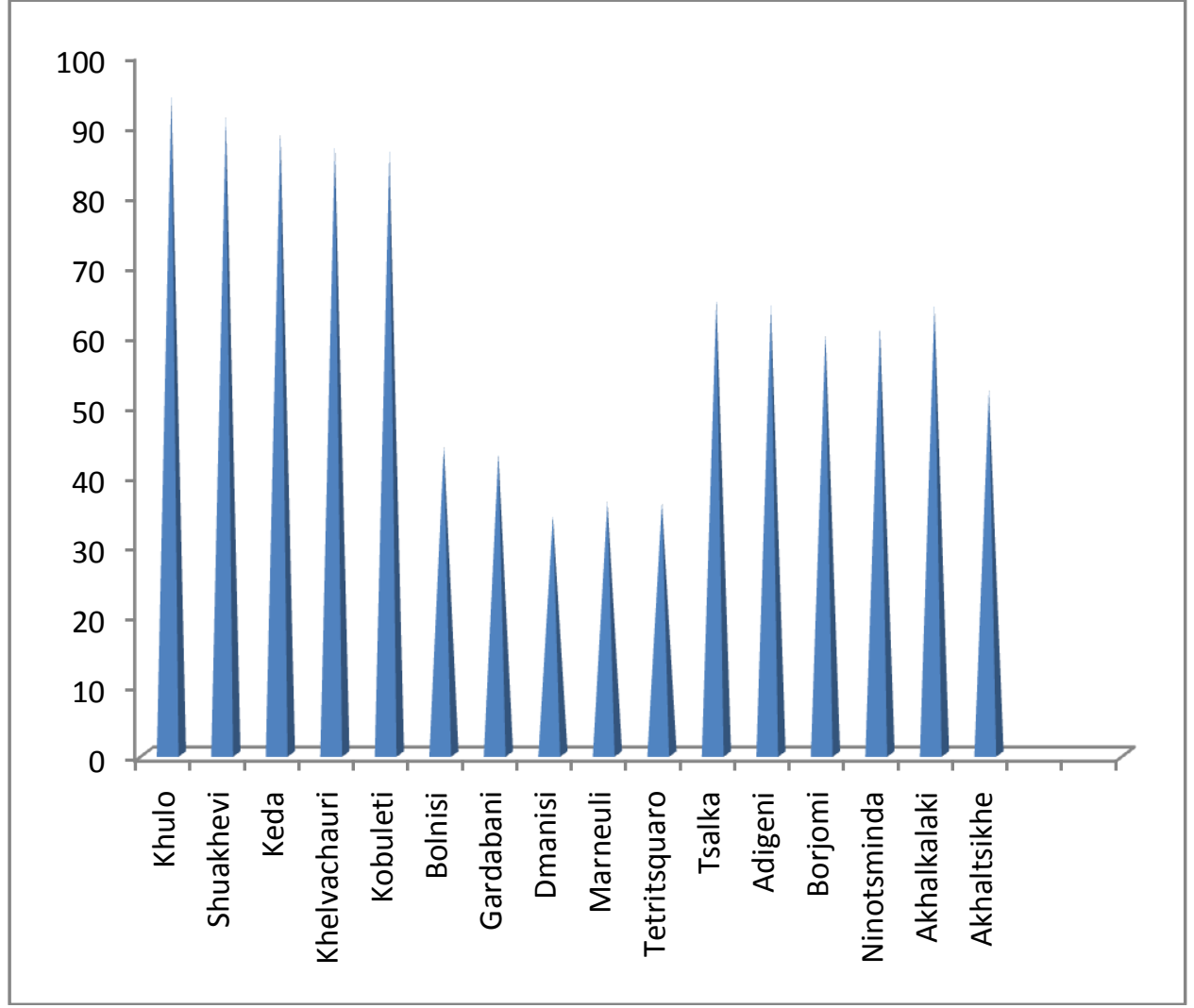


Diagram 4:- Intensity of spreading potato late blight according to regions in Georgia.

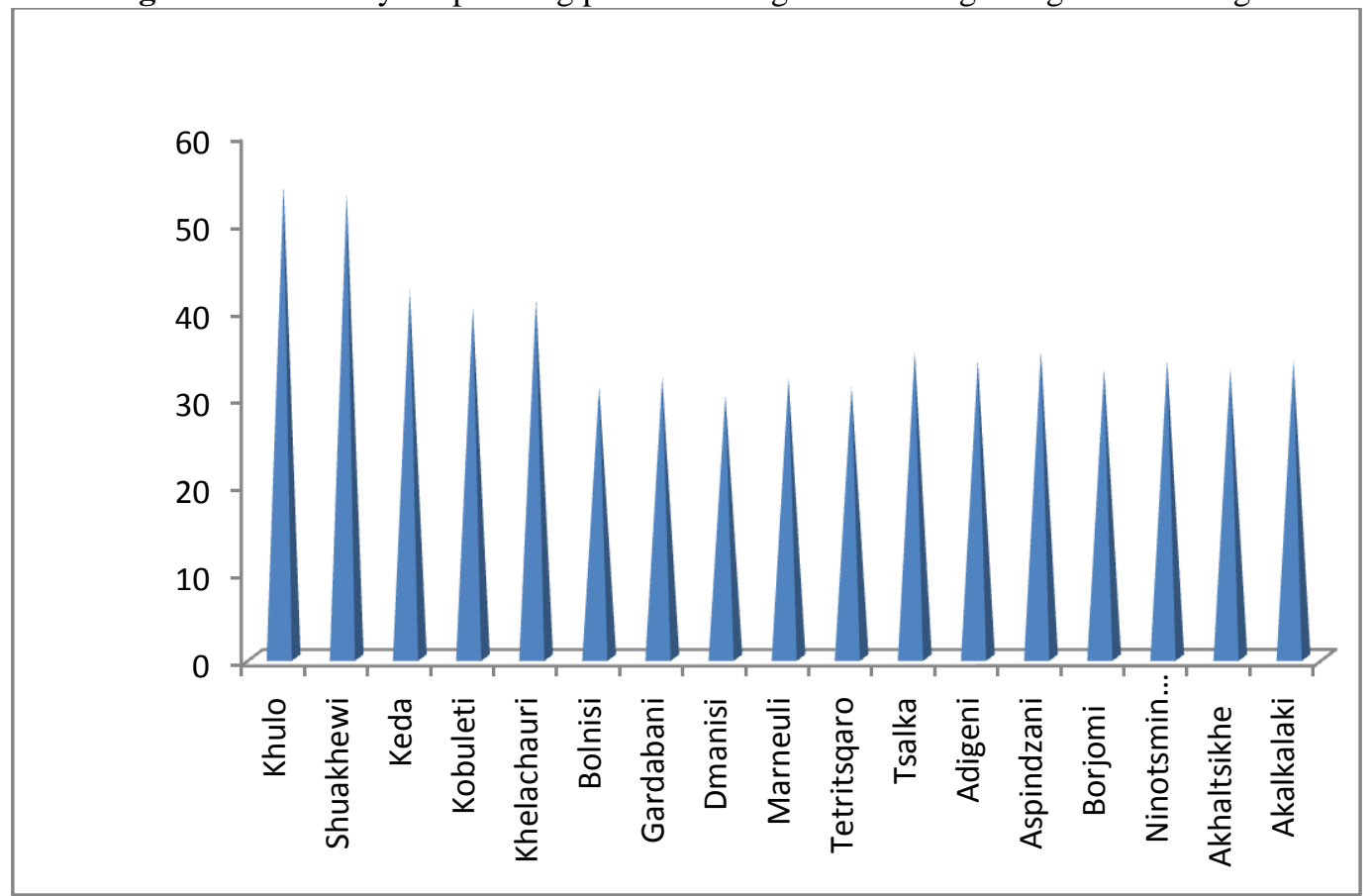

The test of 12 varieties of potatoes (Table 1) shows significant differences in the resistance of varieties. In 20132015 years The highest disease severity was on the Nevsky and Picaso variety, the highest resistance to diseases manifested German variety - Kardena,Russian - Sante, Finland- Katu and Holland variety - Desire.

Increasing the severity of the disease it was noted research years on all grades. In particular, in 2013 the development of late blight was $23.8 \%$, it was $37.7 \%$ in 2014, while it reached $41.4 \%$ in 2015 .

Very high incidence of potato (100\%) was observed in 2015 on Russian variety Nevsi and Picaso.

This year Warm days, and extended wet conditions with rain and fog led to a late blight epidemic, which in less than two weeks, destroyed all the potato.

It was found that every year the loss of potato late blight reached from 5 to 92 percent, depending on location, time of year, weather conditions and cultivars.

Table 1:- Defeat varieties of potato late blight on years, $\%$

\begin{tabular}{|c|c|c|c|c|c|c|c|c|c|}
\hline \multirow{2}{*}{$\begin{array}{l}\text { № } \\
\text { № }\end{array}$} & \multirow{2}{*}{$\begin{array}{l}\text { Potato } \\
\text { varieties }\end{array}$} & \multicolumn{2}{|c|}{2013} & \multicolumn{2}{|c|}{2014} & \multicolumn{2}{|c|}{2015} & \multicolumn{2}{|l|}{ Average } \\
\hline & & $\begin{array}{c}\text { Developme } \\
\text { nt }\end{array}$ & $\begin{array}{c}\text { Spreadin } \\
\mathrm{g}\end{array}$ & Developmen & $\begin{array}{c}\text { Spreadin } \\
\mathrm{g}\end{array}$ & Developmen & $\begin{array}{c}\text { Spreadin } \\
\mathrm{g}\end{array}$ & $\begin{array}{l}\text { Developmen } \\
t\end{array}$ & $\begin{array}{l}\text { Spreadin } \\
\mathrm{g}\end{array}$ \\
\hline$/ 1$ & Arinda & 35,9 & 43,3 & 53,5 & 96,7 & 59,7 & \begin{tabular}{|l|}
97,0 \\
\end{tabular} & 49.7 & 65.9 \\
\hline 2 & Average & 19,8 & 30,0 & 56,7 & 97,7 & 58,2 & 98,4 & 44.9 & 75.4 \\
\hline 3 & Desire & 6,4 & 13,3 & 10,4 & 26,7 & 9,9 & 14,0 & 8.9 & 18.0 \\
\hline 4 & Kardena & 7,7 & 15,8 & 9,2 & 16,8 & 8,5 & 21,2 & 8.46 & 17.9 \\
\hline 5 & Katu & 7,9 & 14,8 & 12,3 & 23,3 & 10,1 & 24,1 & 10.1 & 20.7 \\
\hline 6 & Marfona & 21,8 & 63,3 & 29,6 & 96,7 & 56,7 & 70,0 & 69.4 & 76.7 \\
\hline 7 & Nevski & 46,0 & 64,8 & 63,3 & 95,3 & 76,2 & 100 & 61.8 & 86.7 \\
\hline 8 & Picasso & 45,4 & 64,3 & 63,7 & 94,3 & 76,7 & 100 & 61.9 & 86.2 \\
\hline 9 & Sante & - & - & - & - & 7.8 & 12.6 & 7.8 & 12.6 \\
\hline 10 & Jelly & 41,8 & 73,3 & 69,0 & 56,5 & 50,7 & 70,0 & 53.8 & 66.5 \\
\hline 11 & Wital & 21,2 & 66,6 & 66,7 & 97,7 & 70,7 & 88,0 & 52.7 & 84.1 \\
\hline 12 & Redpantazi & $32,4 \mathrm{H}$ & 49,1 & 18,5 & 83,8 & 15,0 & 61,0 & 23.0 & 64.6 \\
\hline & Average & 23.8 & 41.5 & 37.7 & 65.6 & 41.4 & 63.0 & 32.5 & 56.3 \\
\hline
\end{tabular}




\section{Disease control:-}

Eco-friendly and economically sound tactics to suppress late blight is the immediate goal of many researchers and this objective is also an incentive for a wide range of fundamental research by many scientists. At present, the most reliable approach for integrated management, using an array of tactics, including planting healthy seed tubers, eliminating the source of the pathogen farm, using "resistant" varieties and fungicide application in response to a real need as determined by scouts or forecast.

The biological method used against Phytophtora infestans proved to be the most effective among the agro-technical, sanitary-hygienic, chemical and o ther types of controls. In particular, the antagonist fungus Trichoderma viride (Trichoderma lignorum) was used against pathogens. Soil was tilled with $4 \%$ of the suspension (400 gm of T. viride per $10 \mathrm{Lin} 5 \mathrm{~m}^{2}$ area) prior to planting the potatoes. In this case, the percentage of infection of plant was $7.2 \%$ in a field. The biological efficiency was equal to 83.2. In the control variant, the percentage of infection of plant (without introducing the antagonist into it) was $92.3 \%$. The experimental results have shown that the harvest and economic efficiency respectively are increasing, which were equal to $29.3 \%$ in a field.

\section{Acknowledgements:-}

I thank Guram Aleksidze and anonymous reviewers for helpful suggestions to earlier versions of the manuscript. Additionally I thank previous students, postdoctoral scientists and other colleagues with whom I have been privileged to work over the years.

\section{References:-}

1. Andrivon, D. (1996): The origin of Phytophthora infestans populations present in Europe in the 1840s: a critical review of the historical and scientific evidence. Plant Pathol. 45, 1027-1035.

2. Aylor, D.E., Fry, W.E., Mayton, H. and Andrade-Piedra, J. (2001): Quantifying the rate of release and escape of Phytophthora infestans sporangia from a potato canopy. Phytopathology, 91, 1189-1196.

3. Bilay, V., Elanskaya I. (1982): Methods Experimental Mycology [Journal]. - Kiev: Naukova Dumka, p. 418430.

4. Birch PJ, Bryan G, Fenton B, Gilroy E, Hein I, Jones J, Prashar A, Taylor M, Torrance L, Toth I (2012):

5. Crops that feed the world 8: potato: are the trends of increased global production sustainable? Food Sec 4:477

6. Bourke, A. (1991): Potato late blight in Europe in 1845: the scientific controversy. In: Phytophthora (Lucas,

7. J.A., Shattock, R.D., Shaw, D.S. and Cooke, L.R., eds), New York: Cambridge University Press. pp. 12-24.

8. Dudka, I., Wasser S., Ellanskaya I. (1982): Methods of Experimental Mycology. [Book].- [BM] K. Dumka, p. 4 - 550.

9. Chand, Sudeep (2009): Killer genes cause potato famine, BBC News, retrieved, 09-26.

10. CIP (1996) Enhancing the Global Late Blight Network. Global Initiative on Late Blight. Lima, Peru: Centro Internacional de la Papa.

11. Coffey M.D., Wilson U.E. (1983): Histology and cytology of infection and disease caused by Phytophthora.

12. In Phytophthora, Erwin D.C., Bartnicki-Garcia S., Tsao P.H., eds (St. Paul, MN: American Phytopatholological Society), pp. 289-301.

13. Cvitanich, C. and Judelson, H.S. (2003a): A gene expressed during sexual and asexual sporulation in

14. Phytophthora infestans is a member of the puf family of translational regulators. Eukaryot. Cell, 2, 465-473.

15. Cvitanich, C. and Judelson, H.S. (2003b): Stable ransfomation of the oomycete, Phytophthora infestans,

16. using microprojectile bombardment. Curr. Genet. 42, 228-235.

17. Dowley, L. J., Bannon, E., Cooke, L.R., Keane, T. and O'Sullivan, E. (eds) (1995): Phytophthora infestans,

18. Vol. 150. Dublin: Boole Press Ltd. 7. Fabritius, A.-L., Cvitanich, C. and Judelson, H.S. (2002) Stage-

19. specific gene expression during sexual development in Phytophthora infestans. Mol. Microbiol. 45, 1057- 1066.

20. Fay, J.C. and Fry, W.E. (1997): Effects of hot and cold temperatures on the survival of oospores produced by United States strains of Phytophthora infestans. Am. Potato J. 74, 315-323.

21. Fernandez-Pavia, S.P., Grunwald, N.J., Diaz-Valasis, M., Cadena-Hinojosa, M.A. and Fry, W.E. (2004): Soilborne oospores of Phytophthora infestans in central Mexico survive winter fall and infect potato plants in the field. Plant Dis. 88, 29-33.

22. Flier, W.G., Kroon, L.P.N.M., Hermansen, A., van Raaij, H.M.G., Speiser, B., Tamm, L., Fuchs, J.G., Lambion, J., Razzaghian, J., Andrivon, D., Wilcockson, S. and Leifert, C. (2007):

23. Genetic structure and pathogenicity of populations of Phytophthora infestans from organic potato crops in France, Norway, Switzerland and the United Kingdom. Plant Pathol. 56, 562-572. 
24. Forbes, G.A., Escobar, X.C., Ayala, C.C., Revelo, J., Ordonez, M.E., Fry, B.A., Doucett, K. and Fry, W.E. (1997): Population genetic structure of Phytophthora infestans in Ecuador. Phytopathology, 87, 375-380.

25. Foster, M, Mueller, G., Bills, G. (2004): Biodiversity of fungi. Inventory and monitoring methods [Book]. Boston : Elsevier Academic Press, p. 67- 98.

26. Hawksworth, D. (1974): An introduction to the principles of taxonomy and nomenclature in the fungi and lichens. $[$ Book] = Mycologist's handbook.. - [s.1.] : Kew Commonwealth Mycological Institute, p. 4 - 231.

27. Holmgren, P.K., Holmgren, N.H. and Barbett, L.C. (1990): Index herbariorum, Part. 1: The Herbaria of the World. 8th edn. Regnum vegetabile 120: -163.

28. Khokhryakov, M. (1984): Key crop diseases [Book]. - [S.1.]: L. Kolos,. P. 30-124

29. Garrett, K.A., Nelson, R.J., Mundt, C.C., Chacon, G., Jaramillo, R.E. and Forbes, G.A. (2001): The effects of host diversity and other management components on epidemics of potato late blight in the humid highland tropics. Phytopathology, 91, 993-1000.

Giants L., Sidorov I., Uspenskaya, G. (1980): Field practice on the ecology of fungi and lichens [Magazine]. [BM] M. Univ. University Press, p. 68 - 112.

30. Grunwald, N.J. and Flier, W.G. (2005): The biology of Phytophthora infestans at its center of origin. Annu. Rev. Phytopathol. 43, 171-190.

31. Grunwald, N.J., Romero-Montes, G., Lozoya-Saldana, H., Rubio-Covarrubias, O.A. and Fry, W.E. (2002b): Potato late blight management in the Toluca Valley: field validation of SimCast modified for cultivars with high field resistance. Plant Dis. 86, 1163-1168.

32. Judelson, H.S. and Roberts, S. (2002): Novel protein kinasae induced during sporangial cleavage in the oomycete Phytophthora infestans. Eukaryot. Cell, 1, 687-695.

33. Kriek, N. P. J., T. S. Kellerman, and W. F. 0. Marasas. (1981): A comparative study of the toxicity of Fusarium verticillioides (= F. moniliforme) to horses, primates, pigs, sheep, and rats. Onderstepoort J. Vet. Res. 48:129-131.

34. Kriek, N. P. J., W. F. 0. Marasas, and P. G. Thiel. (1981): Hepato- and cardiotoxicity of Fusarium erticillioides (F. moniliforme) isolates from southern African maize. Food Cosmet. Toxicol. 19:447-456.

35. Kanchaveli, L. A. (1987): Agricultural Phytopathology [Book]. - Tbilisi : [s.n.], 3 - 600.

36. Latijnhouwers, M., Ligterink, W., Vleeshouwers, V.G.A.A., van West, P. and Govers, F. (2004): A Ga

37. subunit controls zoospore motility and virulence in the potato late blight pathogen Phytophthora infestans. Mol. Microbiol. 51, 925-936.

38. Lee, T.Y., Simko, I. and Fry, W.E. (2002): Genetic control of aggressiveness to tomato in Phytophthora infestans. Can. J. Plant Pathol. 24, 471-480.

39. Madden, L.V. and Wheelis, M. (2003): The threat of plant pathogens as weapons against u.s. crops. Annu. Rev. Phytopathol. 41, 155-176.

40. Mizubuti, E.S.G. and Fry, W.E. (2006): Potato late blight. In: The Epidemiology of Plant Diseases (Cooke, B.M., Jones, D.G. and Kaye, B., eds), pp. 445-471.

41. Nowicki, Marcin; et al. (2011): Potato and tomato late blight caused by Phytophthora infestans: An overview of pathology and resistance breeding, Plant Disease, ASP, doi:10.1094.

42. Nowicki, Marcin; et al. (2013): Late blight of tomato. In:Translational Genomics for Crop Breeding: Volume,

43. Biotic Stress, pp.241-265.

44. Pristou R., Gallegly M.E. (1954): Leaf penetration by Phytophthora infestans. Phytopathology 44, 81-86.

45. Shainidze, O. T. (1999): Mycobiota of Adjara [Book]. - Batumi, 5- 355.

46. Shainidze, O. T. (1999): A new species of Micobiota from Adjara [Book]. - Batumi, 3 - 84.

47. Shainidze, O. T. (2013). The Results of Phytopathological Research in Adjara [Book]. - Tbilisi : [s.n.], 3-304

48. Smart, C.D., Mayton, H., Mizubuti, E.S.G., Willmann, M.R. and Fry, W.E. (2000): Environmental and

49. genetic factors influencing self-fertility in Phytophthora infestans. Phytopathology, 90, 987-994.

50. Smart, C.D., Myers, K.L., Restrepo, S., Martin, G.B. and Fry, W.E. (2003): Partial resistance of tomato to Phytophthora infestans is not dependent upon ethylene, jasmonic acid or salicyclic acid signalling pathways. Mol. Plant-Microbe Interact. 16, 141-148.

51. Stewart, H.E., Bradshaw, J.E. and Pande, B. (2003): The effect of the presence of R-genes for resistance to late blight (Phytophthora infestans) of potato (Solanum tuberosum) on the underlying level of field resistance. Plant Pathol. 52, 193-198.

52. Tani, S. and Judelson, H. (2006): Activation of Zoosporogenesis-Specific Genes in Phytophthora infestans Involves a 7-Nucleotide Promoter Motif and Cold-Induced Membrane Rigidity. Eukaryotic Cell, 5 (4), 745752 . 
53. Turner, R.S. (2005): After the famine: plant pathology, Phytophthora infestans and the late blight of potatoes, 1845-1960. Hist. Stud. Phys. Biol. Sci. 34.2, 341-370.

54. Turkensteen, L.J., Flier, W.G., Wanningen, R. and Mulder, A. (2000): Production, survival and infectivity of oospores of Phytophthora infestans. Plant Pathol. 49, 688-696.

55. Vleeshouwers, V.G.A.A., van Dooijeweert, W., Govers, F., Kamoun, S. and Colon, L.T. (2000) The hypersensitive response is associated with host and nonhost resistance to Phytophthora infestans. Planta, 210, 853-864.

56. Whisson, S.C., Avrova, A.O., Van West, P. and Jones, J.T. (2005): A method for double-stranded RNAmediated transient gene silencing in Phytophthora infestans. Mol. Plant Pathol. 6, 153-163.

57. Whisson, S.C., Boevink, P.C., Moleleki, L., Avrova, A.O., Morales, J.G., Gilroy, E.M., Armstrong, M.R.,

58. Grouffaud, S., van West, P., Chapman, S., Hein, I., Toth, I.K., Pritchard, L. and Birch, P.R.J. (2007): A translocation signal for delivery of oomycete effector proteins into host plant cells. Nature, 450, 115-118.

59. Watanabe, T. (2000): Pictorial atlas of soil and seed fungi: Morfologies of cultured fungi and key to species, Florida, p.4-411.

60. Widmark, A.K., Andersson, B., Cassel-Lundhagen, A., Sandstrom, M. and Yuen, J.E. (2007): Phytophthora infestans in a single field in southwest Sweden early in spring: symptoms, spatial distribution and genotypic variation. Plant Pathol. 56, 573-579. 